\title{
Validation of Reference Genes for Gene Expression Normalization in RAW264.7 Cells under Different Conditions
}

\author{
Zhenzhen Bao, ${ }^{1,2}$ Yanli Huang, ${ }^{2}$ Jiyu Chen, ${ }^{2}$ Zhenglong Wang, \\ Jiang Qian, ${ }^{2}$ Jiyang Xu $\mathbb{D}^{2},{ }^{2}$ and Yucheng Zhao $\mathbb{D}^{2,3}$ \\ ${ }^{1}$ School of Pharmacy, Jiangsu Health Vocational College, Nanjing, Jiangsu, China \\ ${ }^{2}$ School of Life Science and Technology, China Pharmaceutical University, Nanjing, Jiangsu, China \\ ${ }^{3}$ Jiangsu Key Laboratory of Bioactive Natural Product Research and State Key Laboratory of Natural Medicines, \\ School of Traditional Chinese Pharmacy, China Pharmaceutical University, Nanjing, Jiangsu, China \\ Correspondence should be addressed to Jiyang Xu; jiyangx@126.com and Yucheng Zhao; zhaoyucheng1986@126.com
}

Received 6 November 2018; Revised 3 March 2019; Accepted 3 April 2019; Published 16 May 2019

Academic Editor: Salvatore Gallone

Copyright (C) 2019 Zhenzhen Bao et al. This is an open access article distributed under the Creative Commons Attribution License, which permits unrestricted use, distribution, and reproduction in any medium, provided the original work is properly cited.

\begin{abstract}
RAW264.7 is a macrophage strain derived from mice tumour and shows a significant ability in antigen uptake. Real-time quantitative PCR (RT-qPCR) is one of the most commonly used methods in gene studies and requires suitable reference genes to normalize and quantitate the expression of gene of interest with sensitivity and specificity. However, suitable reference genes in RAW264.7 cells have not yet been identified for accurate gene expression quantification. In the current study, we evaluated expression levels of ten candidate reference genes in RAW264.7 cells under different conditions. RT-qPCR results indicated significant differences in the expression levels among the ten reference genes. Statistical analyses were carried out using geNorm, NormFinder, and BestKeeper software to further investigate the stability of the reference genes. Integrating the results from the three analytical methods, cytochrome c- 1 and hydroxymethylbilane synthase were found to be the most stable and therefore more suitable reference genes, while ribosomal protein L4 and cyclophilin A were the least stable. This study emphasises the importance of identifying and selecting the most stable reference genes for normalization and provides a basis for future gene expression studies using RAW264.7 cells.
\end{abstract}

\section{Introduction}

Reverse transcription quantitative real-time PCR (RT-qPCR) is an important method for gene expression studies $[1,2]$. This technology has become a very popular method owing to its high speed, high sensitivity, and high-throughput capabilities [3-5]. However, the results are inevitably affected by sample variation and PCR efficiency, which could lead to erroneous interpretations. Hence, in order to ensure accurate measurement of the expression levels of genes in various conditions, normalization of target gene expression with that of a proper reference gene is absolutely necessary when using RT-qPCR [6-8]. Several reference genes, including glyceraldehyde-3phosphate dehydrogenase (GAPDH), beta-2-microglobulin (B2M), and $\beta$-actin (ACTB), have been reported for their stable expression in all tissues and cells $[9,10]$. Considering that gene expression levels may vary among cells or tissues and may also change under certain circumstances, geNorm [11], NormFinder [12], and BestKeeper [13] analytical software have been specially designed for screening of reference gene stability.

Macrophages are important immune cells and play a critical role in anti-infection, antitumour, and immune regulation processes. A large number of cell lines of murine macrophages, such as P338D1 and J774A1 cells, have been commonly used in the study of microbiology and immunology [14, 15]. In contrast, RAW264.7 cells, macrophages derived from mice tumour, have rarely been examined for suitable candidate reference gene expression in a variety of drug treatments [16]. Hence, in this study, we chose RAW264.7 cells to evaluate the expression levels of reference genes for reliable normalization under different conditions. Conversely, a number of studies have shown that no single reference gene exists that can be expressed stably under any 
TABLE 1: Information of the ten reference genes used in the real time quantitative PCR.

\begin{tabular}{|c|c|c|c|c|}
\hline Gene & Description & Primer: forward/reverse $\left(5^{\prime}-3^{\prime}\right)$ & Length (bp) & Accession number \\
\hline \multirow{2}{*}{ ACTB } & \multirow{2}{*}{$\beta$-actin } & F: CAGGTCATCACTATTGGCAA & \multirow{2}{*}{143} & \multirow{2}{*}{ NM_007393 } \\
\hline & & R: AGGTCTTTACGGATGTCAAC & & \\
\hline \multirow{2}{*}{ GAPDH } & \multirow{2}{*}{ Glyceraldehyde-3-phosphate dehydrogenase } & F: TGCTGAGTATGTCGTGGAGT & \multirow{2}{*}{136} & \multirow{2}{*}{ NM_001289726 } \\
\hline & & R:GTTCACACCCATCACAAACA & & \\
\hline \multirow{2}{*}{ RPL4 } & \multirow{2}{*}{ Ribosomal protein L4 } & F: GGAAGTTGGATGAGCTGTAT & \multirow{2}{*}{108} & \multirow{2}{*}{ NM_024212 } \\
\hline & & R: TCAAGATTCTGCTAAGGTCG & & \\
\hline \multirow{2}{*}{ HPRT1 } & \multirow{2}{*}{ Hypoxanthine phosphoribosyltransferase-1 } & F: TAGTGAAACTGGAAAAGCCA & \multirow{2}{*}{135} & \multirow{2}{*}{ NM_013556 } \\
\hline & & R: AAGCTTTACTAGGCAGATGG & & \\
\hline \multirow{2}{*}{ PPIA } & \multirow{2}{*}{ Cyclophilin A } & F:CGGTTCCCAGTTTTTTATCT & \multirow{2}{*}{102} & \multirow{2}{*}{ NM_008907 } \\
\hline & & R: ATGGCTTCCACAATGTTCAT & & \\
\hline \multirow{2}{*}{ CYC1 } & \multirow{2}{*}{ Cytochrome c-1 } & F: CTAACCCTGAGGCTGCAAGA & \multirow{2}{*}{113} & \multirow{2}{*}{ NM_025567 } \\
\hline & & R: GCCAGTGAGCAGGGAAAATA & & \\
\hline \multirow{2}{*}{ HMBS } & \multirow{2}{*}{ Hydroxymethyl-bilane synthase } & F: ATGGCTCAGATAGCATGCAA & \multirow{2}{*}{126} & \multirow{2}{*}{ NM_013551 } \\
\hline & & R: GGGCTCCTCTTGGAATGTTA & & \\
\hline \multirow{2}{*}{ Eeflal } & \multirow{2}{*}{ Eukaryotic translation elongation factor 1 alpha 1} & F: CCTACCACCAACTCGTCCAA & \multirow{2}{*}{136} & \multirow{2}{*}{ NM_010106 } \\
\hline & & R: AAAGGTAACCACCATGCCAG & & \\
\hline \multirow{2}{*}{ GUSB } & \multirow{2}{*}{ Glucuronidase $\beta$} & F: ATGTCCTGCTGAGAGGTGTC & \multirow{2}{*}{121} & \multirow{2}{*}{ NM_010368 } \\
\hline & & R: TCCAGCCTCTCACCAGTAGC & & \\
\hline LDHA & lactate dehydrogenase A & F: ААСАТСТСАСТССССАСАGC & 128 & NM_010699 \\
\hline & 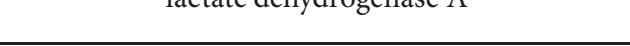 & R: CTCACAGGGGTAATCGAAGC & & 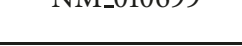 \\
\hline
\end{tabular}

experimental condition [17]. Therefore, ten reference genes, ACTB, GAPDH, ribosomal protein L4 (RPL4), hypoxanthine phosphoribosyltransferase-1 (HPRT1), cyclophilin A (PPIA), Cytochrome c-1 (CYC1), hydroxymethylbilane synthase (HMBS), eukaryotic translation elongation factor 1 alpha 1 (Eefla1), glucuronidase $\beta$ (GUSB), and lactate dehydrogenase A (LDHA), were selected and analysed for suitability under different conditions [9, 10, 18-24].

This study utilized the three analytical methods mentioned above to examine the stability of multiple commonly used reference genes in RAW264.7 cells. To the best of our knowledge, this is the first study to systematically evaluate the expression stability of candidate reference genes in RAW264.7 cells under different conditions (various drugs and concentrations). We believe that the present work will provide a substantial foundation for future research in mouse or human cells.

\section{Materials and Methods}

2.1. Cell Culture and Treatment. Murine macrophage cells, RAW264.7, were grown in DMEM (Gibco, USA) supplemented with $100 \mathrm{U} / \mathrm{mL}$ penicillin-streptomycin and $10 \%$ foetal bovine serum (FBS, Bioind) and incubated at $37^{\circ} \mathrm{C}$ in a $5 \% \quad \mathrm{CO}_{2}$ humidified atmosphere. Upon confluence, cells were trypsinised and treated, respectively, with various drugs at multiple concentrations: highglucose (HG; $50 \mathrm{mM}, 100 \mathrm{mM}, 200 \mathrm{mM}$ ), hydrogen peroxide $\left(\mathrm{H}_{2} \mathrm{O}_{2} ; 50 \mu \mathrm{M}, 100 \mu \mathrm{M}, 200 \mu \mathrm{M}\right)$, lipopolysaccharide (LPS; $0.1,0.5,1 \mu \mathrm{g} / \mathrm{mL})$, cobalt chloride $\left(\mathrm{CoCl}_{2} ; 50 \mu \mathrm{M}\right.$, $100 \mu \mathrm{M}, 200 \mu \mathrm{M})$, and palmitic acid (PA; $50 \mu \mathrm{M}, 100 \mu \mathrm{M}$, $200 \mu \mathrm{M}$ ). Cells without treatment (WT) acted as the control group. Three biological repeats were used for each condition.

2.2. PCR Primers for Reference Genes. Ten reference genes (ACTB, GAPDH, RPL4, HPRT1, PPIA, CYC1, HMBS, Eefla1, GUSB, and LDHA) of RAW264.7 cells were selected for evaluation based on the high frequency of their use in other related studies $[9,10,18-24]$. Primers used for determining the expression of the ten reference genes are listed in Table 1. According to the manufacturer, these primers were designed and optimized using Primer 5 as follows: primer length, 18-22 bp; GC content, 40\%-60\%; amplification length, $100-150 \mathrm{bp}$, to avoid the formation of primerdimer during the reaction.

2.3. RNA Extraction and cDNA Synthesis. RNA was extracted from the treated and control RAW264.7 cells using RNAiso Plus total RNA kit (TransGen Biotech, Dalian, China). DNase I (Takara, Dalian, China) treatment was carried out to purify RNA. The RNA samples with $\mathrm{OD}_{260} / \mathrm{OD}_{280}$ ratios between 1.8 and 2.0 and a total amount of $0.5 \mu \mathrm{g}$ were used for cDNA synthesis to ensure the precision of the trial. Additionally, the purification of the RNA was confirmed by agarose gel electrophoresis. The cDNA was synthesised from the RNA using reverse transcriptase and the HiScript ${ }^{\circledR} \mathrm{Q}$ RT SuperMix for qPCR Kit (Vazyme, Nanjing, China), by following manufacturer's instructions, and stored at $-20^{\circ} \mathrm{C}$ until use for subsequent reaction.

2.4. Quantitative Real-Time PCR Analysis. The RT-qPCR reactions were set up using $C D N A$, forward/reverse primer, 
and Hieff ${ }^{\mathrm{TM}}$ qPCR SYBR ${ }^{\circledR}$ Green Master Mix (Yeasen, Shanghai, China), according to manufacturer's instructions. The reactions were carried out in 96-well PCR reaction plates in a LightCycler 480 system (Roche Molecular Biochemicals, Mannheim, Germany).

2.5. Data Analysis. The stability of reference genes was analysed using the three statistical software programs: geNorm [11], NormFinder [12], and BestKeeper [13]. The geNorm measures gene expression stability according to the values of $\mathrm{M}$, which were calculated from the cycle threshold $(\mathrm{Ct})$ values obtained from RT-qPCR. M values indicated the pairwise variation between individual gene and the other reference genes; lower values of $M$ represented higher expression stabilities, while a higher $M$ value indicated a more unstable reference gene. In addition, geNorm could also determine the optimal number of candidate reference genes required for normalization based on pairwise variation. Like geNorm, NormFinder tended to rank the stability of reference genes according to the $M$ values, with the lowest $M$ value indicating the most stable gene. BestKeeper, an Excel-based statistical method that analyses expression variability of reference genes, ranked the stability of reference genes from most to least based on the key factors of standard deviation (SD) and coefficient of variance $(\mathrm{CV})$.

\section{Results}

3.1. Validation of Primer Specificity. As shown in Supplementary Figure 1, PCR and subsequent agarose gel electrophoresis were used to identify the specificity of the designed primers. The single band and peak of melting curve indicated that the primers possessed good specificity (Supplementary Figure 2).

3.2. The Expression Levels of the Reference Genes. The Ct value generated from the RT-qPCR is the fluorescence threshold for each primer pair and reflects the expression levels of the reference genes; a low Ct value indicates high expression [46]. The overall mean Ct values for the ten reference genes are shown in Figure 1. Among the ten genes, there was an obvious difference in expression levels as seen from the Ct values, which ranged from 15 to 30 . CYC1 was the least expressed reference gene with the highest mean Ct value, while Eeflal, GAPDH, and ACTB were the three most stably expressed genes having lower $\mathrm{Ct}$ values. In addition, LDHA had a narrow range of $\mathrm{Ct}$ values, indicating that the variability of the expression level was constant under different conditions and it might be the best reference gene with a relatively high expression level. Similarly, ACTB and HMBS could be considered as suitable choices for stable reference genes owing to their relatively narrow Ct ranges. In contrast, RPL4 showed a large range of $\mathrm{Ct}$ values and would be unsuitable as a reference gene. To further systematically assess the stability of the ten reference genes under various treatments, the $\mathrm{Ct}$ values were transformed to analyse expression levels using the three software programs: geNorm, NormFinder, and BestKeeper.

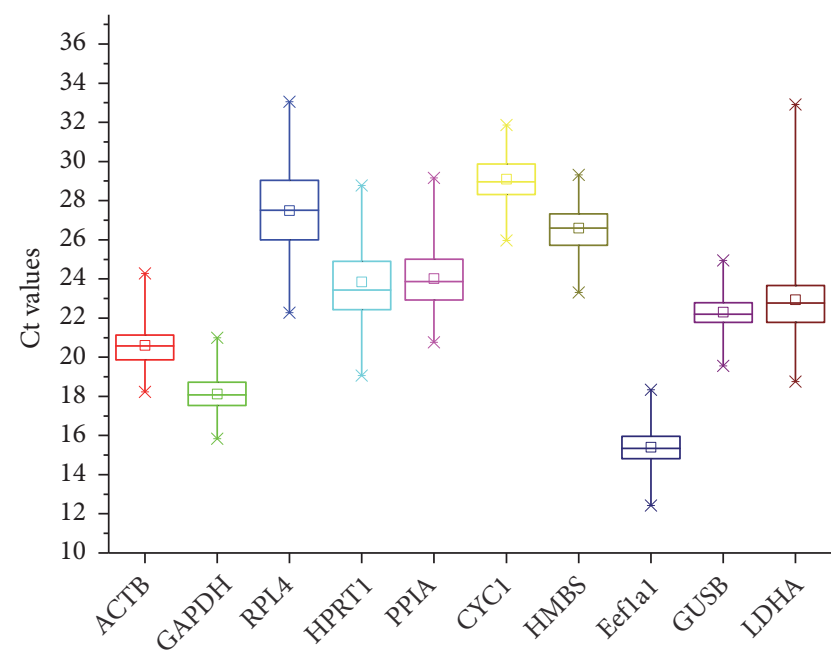

Figure 1: Expression levels of the ten reference genes (ACTB, GAPDH, RPL4, HPRT1, PPIA, CYC1, HMBS, Eeflal, GUSB, and LDHA) in RAW264.7 cells. Squares of the box indicate the means; the lines indicate the median and whiskers indicate the highest and lowest values.

3.3. GeNorm Analysis. GeNorm used the equation $2^{-\Delta \Delta C T}$ (Ct values were collected from different experimental conditions), to transform the $\mathrm{Ct}$ values into relative quantification data to analyse the stability of reference genes, which were ranked from the most to the least stable based on their $M$ values. The $M$ values were calculated by pairwise variation analysis and were considered reliable to select stable reference genes when the $M$ values were greater than 1.5. A lower $M$ value reflected a higher stability [11]. As shown in Figure 2, the stability rank of the ten reference genes was different under different conditions, indicating that different concentrations or drug treatments affected the gene expression. For the control group, the $\mathrm{M}$ values of the ten reference genes were ranked as follows: RPL4 $>$ LDHA > PPIA > HPRT1 > GUSB $>$ ACTB $>$ HMBS $>$ Eeflal $>\mathrm{CYC1}>\mathrm{GAPDH}$, revealing that GAPDH was the most stable reference gene, while RPL4 was the least stable. However, for other groups, the ten reference genes had different stability ranks. For instance, the rank of the reference genes after LPS treatment $(0.5 \mu \mathrm{g} / \mathrm{mL})$ was as follows: PPIA > LDHA > ACTB > GUSB > RPL4 > Eeflal > $\mathrm{CYC1}>\mathrm{HMBS}>\mathrm{GAPDH}>\mathrm{HPRT} 1$, indicating that, under these conditions, HPRT1 ranked the most stable reference gene, while PPIA was the least stable. In addition, different concentrations of the same drug resulted in the same gene to be ranked as the most stable reference gene; for example, ACTB was the most stable reference gene after treatment with both low and high concentrations of HG. Moreover, different drug treatments showed the same reference gene to be the most stable at similar doses of treatment. For example, HMBS was ranked the most stable candidate gene in $\mathrm{H}_{2} \mathrm{O}_{2}$, LPS, and $\mathrm{CoCl}_{2}$ treatments, respectively, but only upon treatment with high concentrations of the drugs. Taken together, these results indicated that the stability of the reference genes was not constant under different conditions. Notably, geNorm analysis indicated that GAPDH might be the most stable 

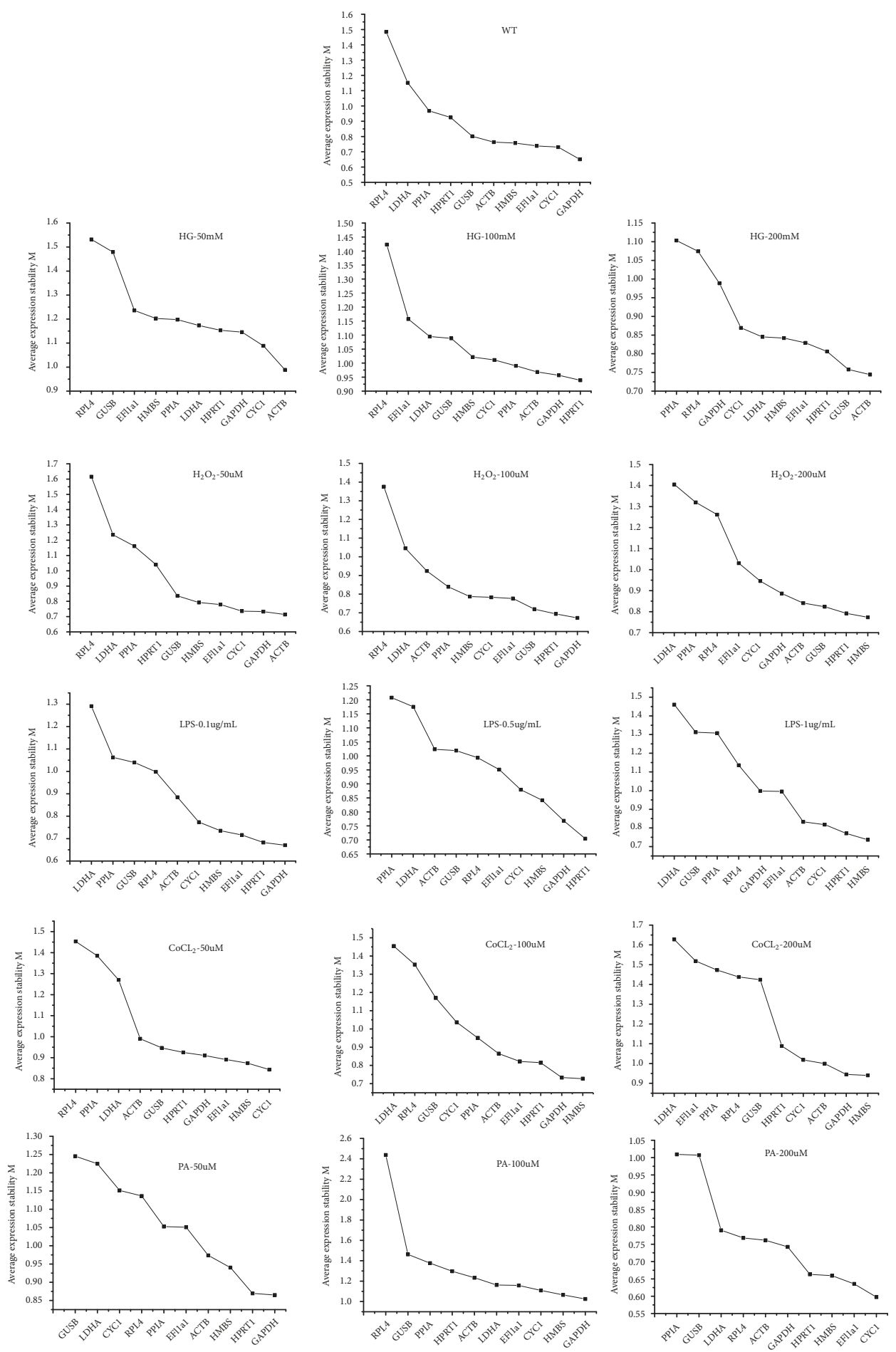

FIGURE 2: Expression stability of the reference genes analysed by geNorm. M values represent the average expression stability; the stability is ranked from left to right, indicating the stability is inversely related to the $\mathrm{M}$ values. The treatments of drugs and concentrations are listed in the figure correspondingly.

reference genes under various experimental conditions (drug treatments and concentrations).

3.4. NormFinder Analysis. The NormFinder is a statistical algorithm that calculates the $M$ values, which are then used to rank the stability of the reference genes. Similar to geNorm, the most stable gene is associated with the smallest $M$ value [12]. As shown in Table 2, the ten reference genes in the control group were ranked based on stability as follows: GAPDH, HMBS, CYC1, GUSB, ACTB, HPRT1, 


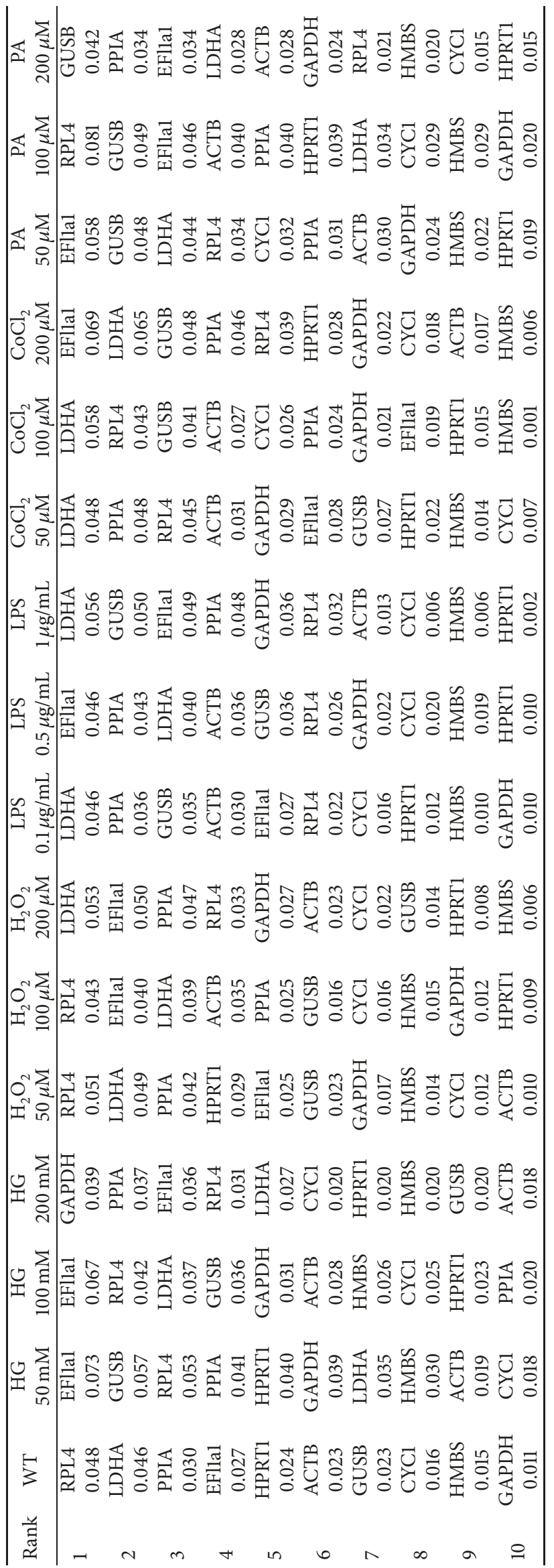




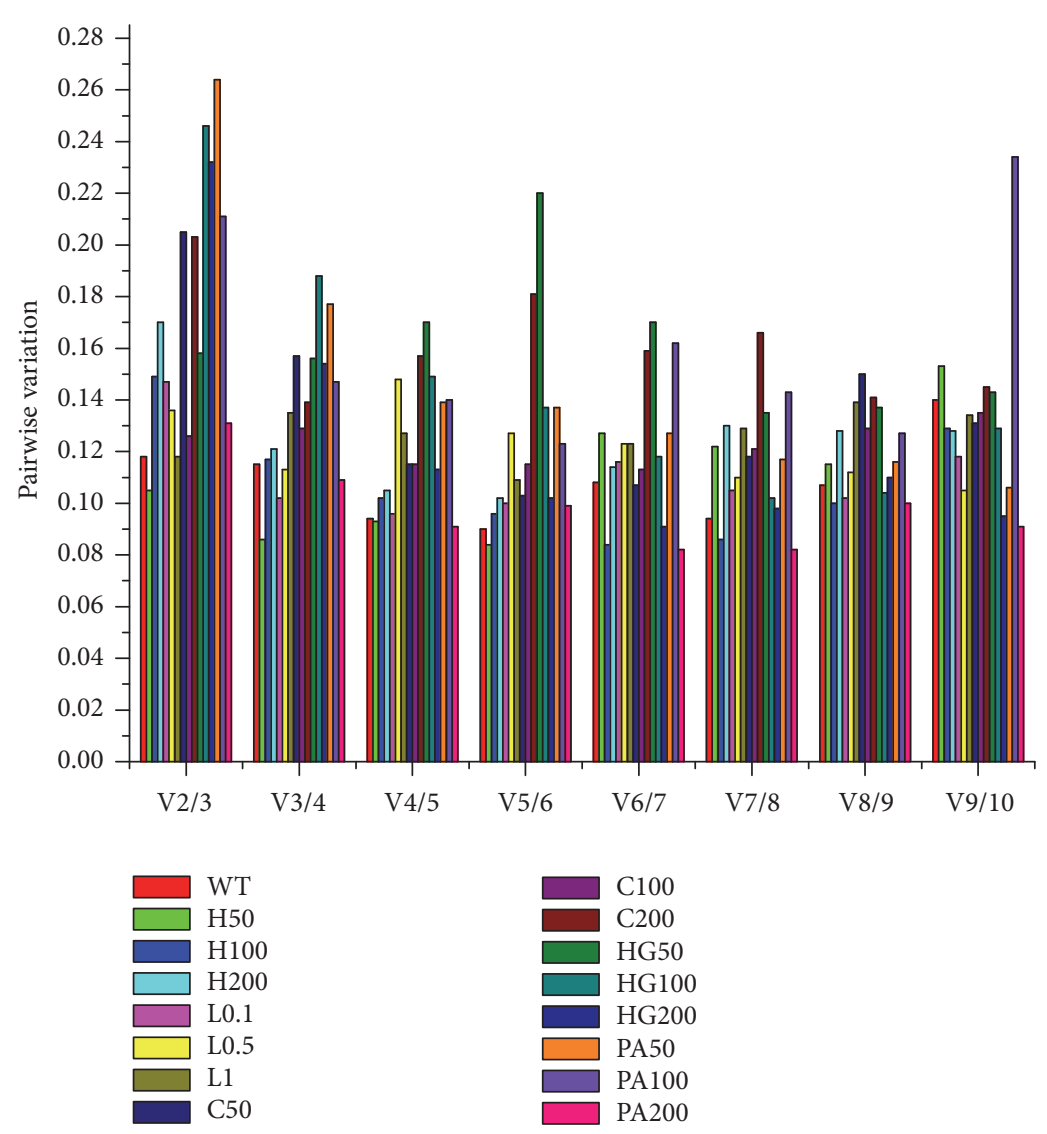

FIGURE 3: Determination of the optimal number of reference genes for normalization using geNorm analysis. Pairwise variation $(\mathrm{Vn} / \mathrm{n}+1)$ of reference genes under different conditions are listed. WT, H50 (H100, H200), L0.1 (L0.5, L1), C50 (C100, C200), HG50 (HG100, HG200), and PA50 (PA100, PA200), respectively, were the abbreviation for the control group; $\mathrm{H}_{2} \mathrm{O}_{2}, 50 \mu \mathrm{M}(100 \mu \mathrm{M}, 200 \mu \mathrm{M})$; lipopolysaccharide, $0.1 \mu \mathrm{g} / \mathrm{mL}(0.5,1 \mu \mathrm{g} / \mathrm{mL})$; $\mathrm{CoCl}_{2}, 50 \mu \mathrm{M}(100 \mu \mathrm{M}, 200 \mu \mathrm{M})$; high-glucose, $50 \mathrm{mM}(100 \mathrm{mM}, 200 \mathrm{mM})$; and palmitic acid, $50 \mu \mathrm{M}(100 \mu \mathrm{M}$, $200 \mu \mathrm{M})$.

Eeflal, PPIA, LDHA, and RPL4. Thus, GAPDH was the most expressed stable reference gene, followed by HMBS; RPL4 was the least expressed one. These results were similar to those obtained with geNorm data analysis of the control group. Interestingly, under some conditions, more than one reference gene showed the same $M$ value, and therefore identical stability; for instance, CYC1 and HPRT1 showed the same $M$ value and were determined to be the most stably expressed genes upon treatment with $200 \mu \mathrm{M}$ PA. Consistent with the results of geNorm analysis, the HMBS gene seemed to be the most stable reference gene, as it showed the lowest $\mathrm{M}$ value among the most stable genes under different conditions. However, differences were also noted between the geNorm and NormFinder analyses; for instance, CYC1 was ranked as the most stable gene by NormFinder, while geNorm ranked it as the fourth.

3.5. BestKeeper Analysis. Distinct from the geNorm and NormFinder methods, BestKeeper determines the gene stability based on the SD and the CV. The SD of the reference genes is a key factor to identify the most stable gene in the BestKeeper analysis; the higher the CV and SD values, the more unstable the reference gene [13]. Supplementary Table 1 shows the $\mathrm{CV} \pm \mathrm{SD}$ values, arranged in the decreasing order of their value from top to bottom, representing the stability rank of the reference gene from least to most. Reference genes with $\mathrm{SD}>1.5$ were eliminated for further analysis. Specifically, PPIA $(\mathrm{SD}=1.53)$ in $50 \mu \mathrm{M} \mathrm{CoCl}$, LDHA $(\mathrm{SD}=1.56)$ in $200 \mu \mathrm{M} \mathrm{CoCl}_{2}$, and RPL4 $(\mathrm{SD}=2.50)$ were excluded to be able to accurately select the best normalization gene from the candidate genes. GUSB and HMBS frequently appeared as the most stable reference genes. In contrast, Eeflal and RPL4 often ranked as the top-most unstable genes. Notably, in the control group, GUSB had the smallest $\mathrm{CV} \pm \mathrm{SD}$ values of $0.34 \pm 0.07$, followed by CYC1 with $1.30 \pm 0.36$, and RPL4 had the highest expression variation with $\mathrm{CV} \pm \mathrm{SD}$ value of $4.13 \pm 1.19$. Together, the above findings indicated that BestKeeper analysis differed significantly from that of geNorm and NormFinder analysis.

3.6. Optimal Number of Reference Genes for Normalization. GeNorm can select the most stable expression gene and also determine the optimal number of reference genes for accurate normalization according to the pairwise variation $(\mathrm{Vn} / \mathrm{n}+1)$, which is calculated from the two sequential normalization factors (NFn) and uses $\mathrm{Vn} / \mathrm{n}+1<0.15$ as a criterion to confirm the optimal number of reference genes for accurate analysis. The results of $V n / n+1$ are shown in Figure 3. Most of the 
$\mathrm{V} 2 / 3$ values were greater than 0.15 , indicating that using only two most stably expressed genes would not improve the accuracy under all conditions. According to the cutoff value of 0.15 , the control group, $\mathrm{H}_{2} \mathrm{O}_{2}(50 \mu \mathrm{M}, 100 \mu \mathrm{M}$, $200 \mu \mathrm{M})$, LPS $(0.1,0.5,1 \mu \mathrm{g} / \mathrm{mL}), 100 \mu \mathrm{M} \mathrm{CoCl}_{2}$, and $200 \mu \mathrm{M}$ PA could be assessed using only two suitable reference genes, while three reference genes were required under conditions of $200 \mu \mathrm{M} \mathrm{CoCl}_{2}$ and $100 \mu \mathrm{M} \mathrm{PA}$, and four were required under conditions of $\mathrm{HG}(50 \mathrm{mM}, 100 \mathrm{mM}, 200 \mathrm{mM}), 50 \mu \mathrm{M} \mathrm{CoCl}$, and $50 \mu \mathrm{M}$ PA.

\section{Discussion}

It is critical to select the proper reference genes for normalization when using RT-qPCR quantification for analysing gene expression [25-27]. Our study evaluated the stability of ten reference genes in RAW264.7 cells by geNorm [11], NormFinder [12], and BestKeeper [13] programs, by comparing their expression levels under different conditions. The $\mathrm{Ct}$ values representing expression levels of the reference genes ranged from 15 to 30 , in accordance with the results of most reports [4].

Based on the principle that lower $\mathrm{Ct}$ values indicate higher expression profile $[4,6]$, our results indicated that Eeflal, GAPDH, and ACTB were the three most highly expressed reference genes, while CYC1 was the least expressed gene with $\mathrm{Ct}$ values being nearly 30 under conditions. In addition, owing to a narrow $\mathrm{Ct}$ range, which represents a stable expression profile, LDHA, ACTB, and HMBS were considered as the most stable reference genes, while RPL4 was the least stable. Our findings showed some differences between geNorm, NormFinder, and BestKeeper analyses, indicating that it is necessary to systematically analyse expression stability combining various analysis methods, as also suggested by previous reports [28-30].

GeNorm analysis indicated that GAPDH, HMBS, and HPRT1 were the three most stable genes under different experimental conditions. NormFinder ranked HMBS, CYC1, HPRTI, and GAPDH on the basis of high expression stability, while BestKeeper indicated that GUSB and HMBS could be considered as the most suitable normalization genes. Thus, the stability ranking of the candidate genes was not consistent across different analyses. Nonetheless, on comparing the outcomes of the three analytical programs, HMBS was the only one that was commonly identified by all three programs to be highly and stably expressed. Additionally, only slight differences were observed between the stability of the ten reference genes when comparing results of NormFinder and geNorm, likely due to the similar method adopted by both programs to evaluate gene expression stability. Integrating results of all three analyses, CYC1 and HMBS were considered to be the most stably expressing genes suitable for normalizing qPCR data in RAW264.7 cells.

GAPDH is a frequently used reference gene, although a number of studies have reported that it does not maintain constant expression level under different experimental conditions, and thus it is unsuitable for normalizing RT-qPCR data [31-34]. However, GAPDH was recognized as the most stable gene with a lower $\mathrm{M}$ value by geNorm analysis in our study, consistent with previous studies in mouse uterus cells and J774A1 murine macrophage cells [15, 35]. RPL4 and PPIA significantly ranked low, indicating that they were not suitable as normalizing genes. However, previous studies have reported that RPL4 was a highly stable gene, as its expression was not influenced by some experimental conditions [3638]. Meanwhile, PPIA has been considered a highly expressed reference gene for normalization in macrophages [33, 37].

Considering that using a single reference gene could lead to inaccuracies during RT-qPCR quantification [39, 40 ], it is necessary to determine the optimal number of reference genes to be used for gene expression studies. In the current study, a pairwise variation $(\mathrm{Vn} / \mathrm{n}+1)$ indicated that most groups required only two reference genes for normalization, as the $\mathrm{V} 2 / 3$ values were lower than the cut-off value of 0.15 . However, the $\mathrm{Vn} / \mathrm{n}+1$ values of certain groups exceeded 0.15 , suggesting the need for additional reference genes (three or four) to improve the accuracy of normalization.

\section{Conclusions}

In summary, we evaluated the stability of ten reference genes in RAW264.7 cells using three analytical approaches, namely, geNorm, NormFinder, and BestKeeper, to determine the most stable reference genes under different conditions. Our findings reveal that two reference genes are sufficient for accurate normalization in most conditions, although some cases might require more than two reference genes for accurate evaluation of gene expression levels. Importantly, our data indicate that CYC1 and HMBS are the most suitable reference genes, while RPL4 and PPIA are the most variable and unsuitable genes for normalization in RAW264.7 cells. These findings might play a crucial role in the selection of appropriate reference genes in further studies.

\section{Data Availability}

The data used to support the findings of this study are included within the article.

\section{Conflicts of Interest}

The authors declare no conflicts of interest.

\section{Authors' Contributions}

Zhenzhen Bao, Yanli Huang, and Jiyu Chen contributed equally to this work.

\section{Acknowledgments}

This project is funded by National Natural Science Foundation of China (81703637, 81573564), China Postdoctoral Science Foundation (2016M601922, 2018T110577), and Natural Science Fund in Jiangsu Province (BK20170736). This work is also funded by teaching and research project of Jiangsu Health Vocational College (JKKYTD201701, JKA201812). 


\section{Supplementary Materials}

Supplementary 1. Supplementary Figure 1: Agarose gel (1\%) electrophoresis of the ten reference genes. 1-10 represent ACTB, GAPDH, RPL4, HPRT1, PPIA, CYC1, HMBS, Eefla1, GUSB, and LDHA, respectively.

Supplementary 2. Supplementary Figure 2: Melt curves of the ten reference genes.

Supplementary 3. Supplementary Table 1: Expression stability values of the ten reference genes calculated by BestKeeper in RAW264.7 cells.

\section{References}

[1] C. Caldana, W.-R. Scheible, B. Mueller-Roeber, and S. Ruzicic, "A quantitative RT-PCR platform for high-throughput expression profiling of 2500 rice transcription factors," Plant Methods, vol. 3, no. 1, p. 7, 2007.

[2] M. W. Pfaffl, “The ongoing evolution of qPCR," Methods, vol. 50, no. 4, pp. 215-216, 2010.

[3] X. Li, D. Zhang, H. Li et al., "Characterization of reference genes for RT-qPCR in the desert moss Syntrichia caninervis in response to abiotic stress and desiccation/rehydration," Frontiers in Plant Science, vol. 6, p. 38, 2015.

[4] S. A. Bustin, V. Benes, J. A. Garson et al., "The MIQE guidelines: minimum information for publication of quantitative real-time PCR experiments," Clinical Chemistry, vol. 55, no. 4, pp. 611-622, 2009.

[5] H. Cassan-Wang, M. Soler, H. Yu et al., "Reference genes for high-throughput quantitative reverse transcription-PCR analysis of gene expression in organs and tissues of eucalyptus grown in various environmental conditions," Plant \& Cell Physiology (PCP), vol. 53, no. 12, pp. 2101-2116, 2012.

[6] Q. Gao, X.-Y. Wang, J. Fan et al., "Selection of reference genes for real-time PCR in human hepatocellular carcinoma tissues," Journal of Cancer Research and Clinical Oncology, vol. 134, no. 9, pp. 979-986, 2008.

[7] S. M. Gresner, E. Golanska, D. Kulczycka-Wojdala, D. J. Jaskolski, W. Papierz, and P. P. Liberski, "Selection of reference genes for gene expression studies in astrocytomas," Analytical Biochemistry, vol. 408, no. 1, pp. 163-165, 2011.

[8] H. Zhuang, Y. Fu, W. He, L. Wang, and Y. Wei, "Selection of appropriate reference genes for quantitative real-time PCR in Oxytropis ochrocephala Bunge using transcriptome datasets under abiotic stress treatments," Frontiers in Plant Science, vol. 6, p. 475, 2015.

[9] Y. Higashimura, Y. Nakajima, R. Yamaji et al., "Up-regulation of glyceraldehyde-3-phosphate dehydrogenase gene expression by HIF-1 activity depending on Sp1 in hypoxic breast cancer cells," Archives of Biochemistry and Biophysics, vol. 509, no. 1, pp. 1-8, 2011.

[10] F. Wang, J. Wang, D. Liu, and Y. Su, "Normalizing genes for realtime polymerase chain reaction in epithelial and nonepithelial cells of mouse small intestine," Analytical Biochemistry, vol. 399, no. 2, pp. 211-217, 2010.

[11] J. Vandesompele, K. De Preter, F. Pattyn et al., "Accurate normalization of real-time quantitative RT-PCR data by geometric averaging of multiple internal control genes," Genome Biology, vol. 3, no. 7, Article ID RESEARCH0034, 2002.
[12] C. L. Andersen, J. L. Jensen, and T. F. Ørntoft, "Normalization of real-time quantitative reverse transcription-PCR data: a modelbased variance estimation approach to identify genes suited for normalization, applied to bladder and colon cancer data sets," Cancer Research, vol. 64, no. 15, pp. 5245-5250, 2004.

[13] M. W. Pfaffl, A. Tichopad, C. Prgomet, and T. P. Neuvians, "Determination of stable housekeeping genes, differentially regulated target genes and sample integrity: bestKeeper-excelbased tool using pair-wise correlations," Biotechnology Letters, vol. 26, no. 6, pp. 509-515, 2004.

[14] D. M. Segal, S. K. Dower, and J. A. Titus, "The FcR-mediated endocytosis of model immune complexes by cells from the P338D1 mouse macrophage line. I. Internalization of small, nonaggregating oligomers of IgG," The Journal of Immunology, vol. 130, no. 1, pp. 130-137, 1983.

[15] F. Ferraz and J. Fernandez, "Selection and validation of reference house-keeping genes in the J774A1 macrophage cell line for quantitative real-time PCR," Genetics and Molecular Research, vol. 15, no. 1, Article ID 15017720, 2016.

[16] D. L. Taylor, P. C. Thomson, K. de Silva, and R. J. Whittington, "Validation of endogenous reference genes for expression profiling of RAW264.7 cells infected with Mycobacterium avium subsp. paratuberculosis by quantitative PCR," Veterinary Immunology and Immunopathology, vol. 115, no. 1-2, pp. 43-55, 2007.

[17] K. Dheda, J. F. Huggett, J. S. Chang et al., "The implications of using an inappropriate reference gene for real-time reverse transcription PCR data normalization," Analytical Biochemistry, vol. 344, no. 1, pp. 141-143, 2005.

[18] Z. Kolkova, A. Arakelyan, B. Casslén, S. Hansson, and E. Kriegova, "Normalizing to GADPH jeopardises correct quantification of gene expression in ovarian tumours - IPO8 and RPL4 are reliable reference genes," Journal of Ovarian Research, vol. 6, no. 1, p. 60, 2013.

[19] A. S. Stephens, S. R. Stephens, and N. A. Morrison, "Internal control genes for quantitative RT-PCR expression analysis in mouse osteoblasts, osteoclasts and macrophages," $B M C$ Research Notes, vol. 4, no. 1, p. 410, 2011.

[20] O. J. Harrison, N. Moorjani, C. Torrens, S. K. Ohri, and F. R. Cagampang, "Endogenous reference genes for gene expression studies on bicuspid aortic valve associated aortopathy in humans," PLoS ONE, vol. 11, no. 10, Article ID e0164329, 2016.

[21] W. Zhu, Y. Lin, H. Liao, and Y. Wang, "Selection of reference genes for gene expression studies related to intramuscular fat deposition in Capra hircus skeletal muscle," PLoS ONE, vol. 10, no. 3, Article ID e0121280, 2015.

[22] B. R. Everaert, G. A. Boulet, J.-P. Timmermans, and C. J. Vrints, "Importance of suitable reference gene selection for quantitative real-time PCR: special reference to mouse myocardial infarction studies," PLoS ONE, vol. 6, no. 8, Article ID e23793, 2011.

[23] T. Fink, P. Lund, L. Pilgaard, J. G. Rasmussen, M. Duroux, and V. Zachar, "Instability of standard PCR reference genes in adipose-derived stem cells during propagation, differentiation and hypoxic exposure," BMC Molecular Biology, vol. 9, p. 98, 2008.

[24] S. Ren, F. Zhang, C. Li et al., "Selection of housekeeping genes for use in quantitative reverse transcription PCR assays on the murine cornea," Molecular Vision, vol. 16, pp. 1076-1086, 2010.

[25] D. G. Ginzinger, "Gene quantification using real-time quantitative PCR: an emerging technology hits the mainstream," Experimental Hematology, vol. 30, no. 6, pp. 503-512, 2002. 
[26] V. J. Gadkar and M. Filion, "Validation of endogenous reference genes in Buglossoides arvensis for normalizing RT-qPCR-based gene expression data," SpringerPlus, vol. 4, no. 1, p. 178, 2015.

[27] D. V. Maltseva, N. A. Khaustova, N. N. Fedotov et al., "Highthroughput identification of reference genes for research and clinical RT-qPCR analysis of breast cancer samples," Journal of Clinical Bioinformatics, vol. 3, no. 1, p. 13, 2013.

[28] Y. Zhao, J. Luo, S. Xu et al., "Selection of reference genes for gene expression normalization in Peucedanum praeruptorum Dunn under abiotic stresses, hormone treatments and different tissues," PLoS ONE, vol. 11, no. 3, Article ID e0152356, 2016.

[29] F. Almeida-Oliveira, J. G. Leandro, P. Ausina, M. Sola-Penna, and D. Majerowicz, "Reference genes for quantitative PCR in the adipose tissue of mice with metabolic disease," Biomedicine \& Pharmacotherapy, vol. 88, pp. 948-955, 2017.

[30] B. Żyżyńska-Granica and K. Koziak, "Identification of suitable reference genes for real-time PCR analysis of statin-treated human umbilical vein endothelial cells," PLoS ONE, vol. 7, no. 12, Article ID e51547, 2012.

[31] J. Gimeno, N. Eattock, A. Van Deynze, and E. Blumwald, "Selection and validation of reference genes for gene expression analysis in switchgrass (Panicum virgatum) using quantitative real-time RT-PCR," PLoS ONE, vol. 9, no. 3, Article ID e91474, 2014.

[32] P. Matouskova, H. Bartikova, I. Bousova, V. Hanusova, B. Szotakova, and L. Skalova, "Reference genes for real-time PCR quantification of messenger rnas and micrornas in mouse model of obesity," PLoS ONE, vol. 9, no. 1, Article ID e86033, 2014.

[33] F. Jacob, R. Guertler, S. Naim et al., "Careful selection of reference genes is required for reliable performance of RT-qPCR in human normal and cancer cell lines," PLoS ONE, vol. 8, no. 3, Article ID e59180, 2013.

[34] R. D. Barber, D. W. Harmer, R. A. Coleman, and B. J. Clark, "GAPDH as a housekeeping gene: analysis of GAPDH mRNA expression in a panel of 72 human tissues," Physiological Genomics, vol. 21, no. 3, pp. 389-395, 2005.

[35] P. Lin, X. Lan, F. Chen, Y. Yang, Y. Jin, and A. Wang, "Reference gene selection for real-time quantitative PCR analysis of the mouse uterus in the peri-implantation period," PLoS ONE, vol. 8, no. 4, Article ID e62462, 2013.

[36] M. U. Cinar, M. A. Islam, M. Pröll et al., "Evaluation of suitable reference genes for gene expression studies in porcine PBMCs in response to LPS and LTA," BMC Research Notes, vol. 6, no. 1, p. 56, 2013.

[37] S.-J. Park, S. G. Kwon, J. H. Hwang, D. H. Park, T. W. Kim, and C. W. Kim, "Selection of appropriate reference genes for RTqPCR analysis in Berkshire, Duroc, Landrace, and Yorkshire pigs," Gene, vol. 558, no. 1, pp. 152-158, 2015.

[38] C. Zeng, L. He, W. Peng et al., "Selection of optimal reference genes for quantitative RT-PCR studies of boar spermatozoa cryopreservation," Cryobiology, vol. 68, no. 1, pp. 113-121, 2014.

[39] K. E. Reid, N. Olsson, J. Schlosser, F. Peng, and S. T. Lund, “An optimized grapevine RNA isolation procedure and statistical determination of reference genes for real-time RT-PCR during berry development," BMC Plant Biology, vol. 6, p. 27, 2006.

[40] A. R. Paolacci, O. A. Tanzarella, E. Porceddu, and M. Ciaffi, "Identification and validation of reference genes for quantitative RT-PCR normalization in wheat," BMC Molecular Biology, vol. 10, p. 11, 2009. 


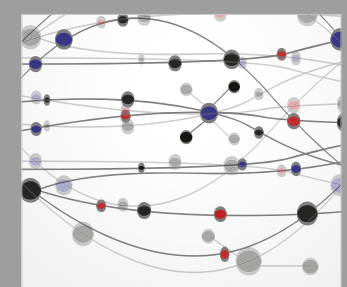

The Scientific World Journal
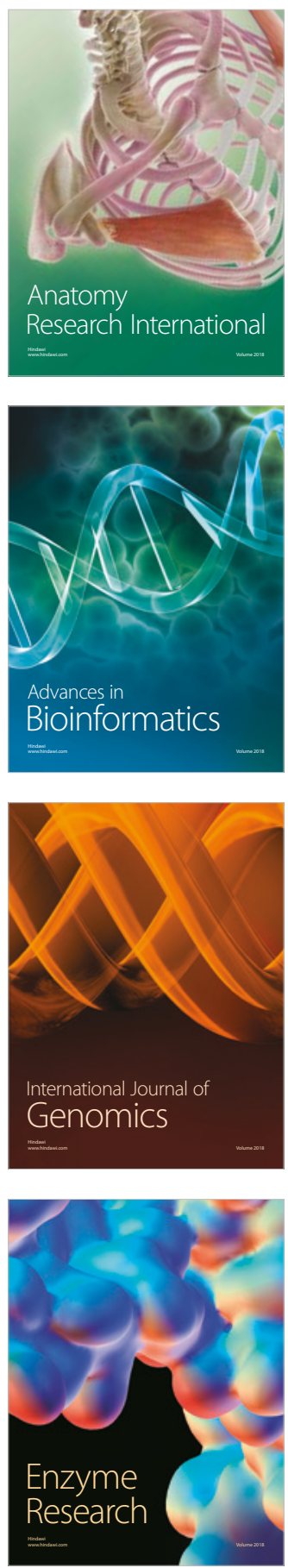
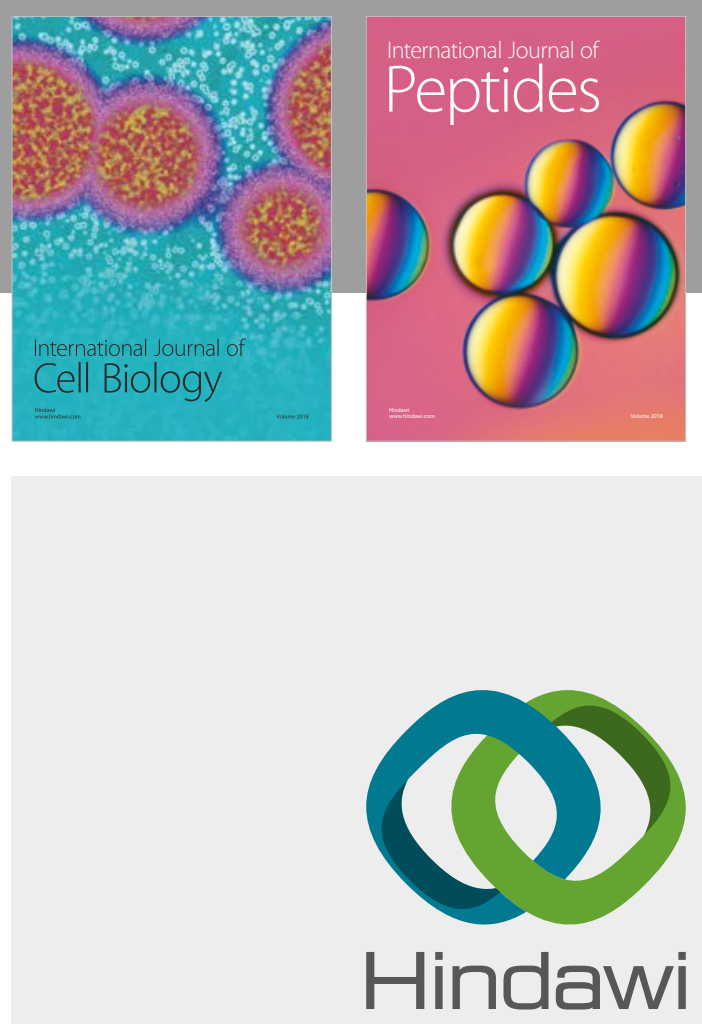

Submit your manuscripts at

www.hindawi.com
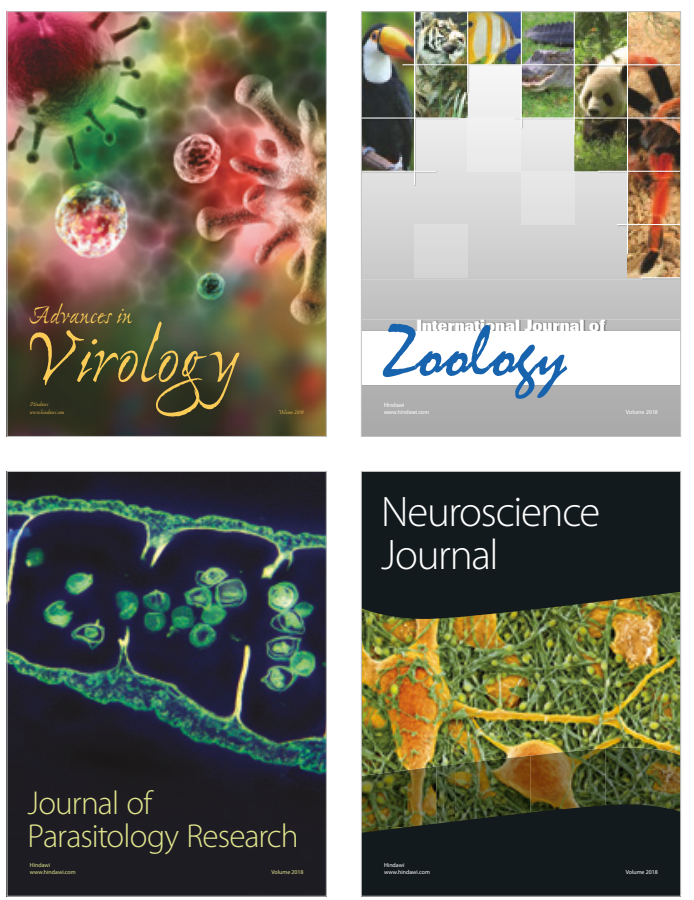
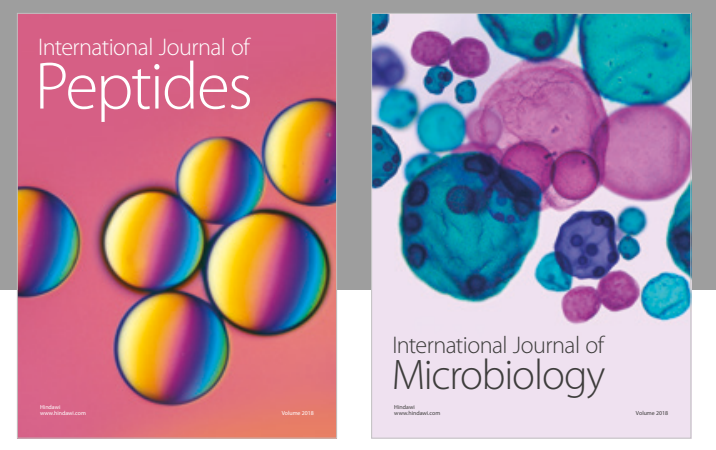

nternational Journal of Microbiology
Journal of
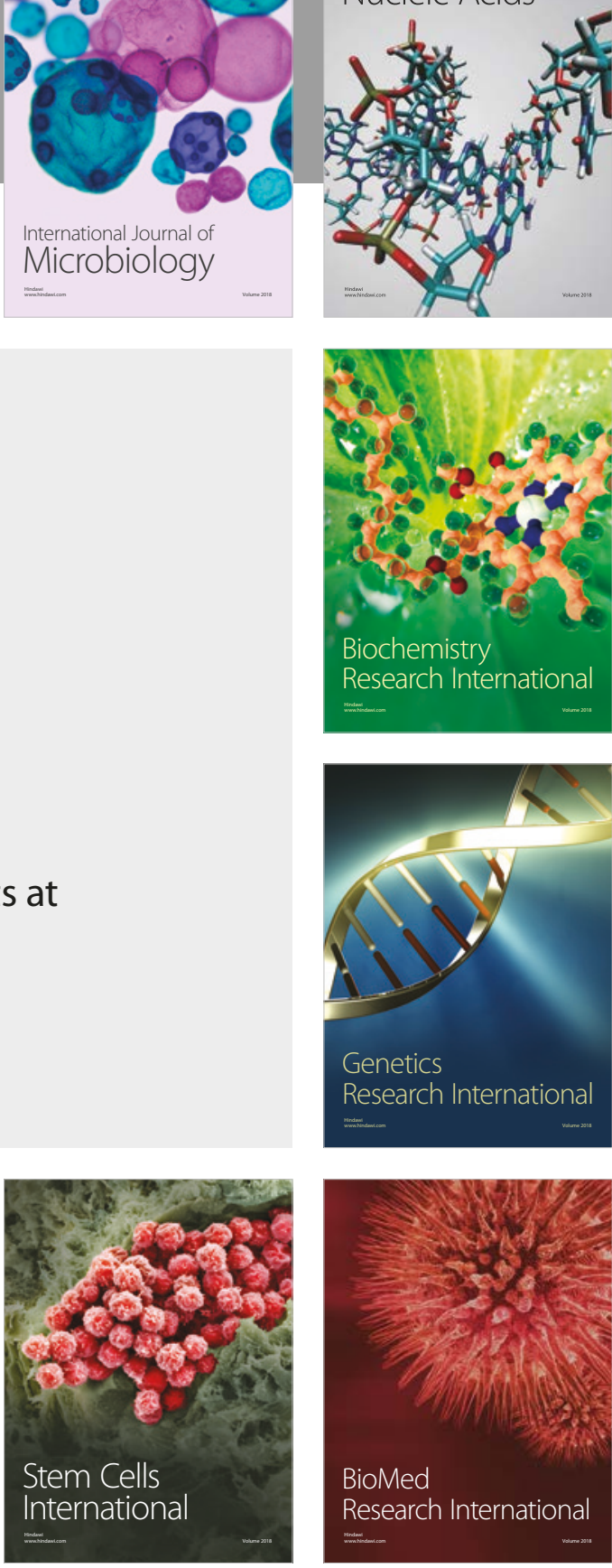
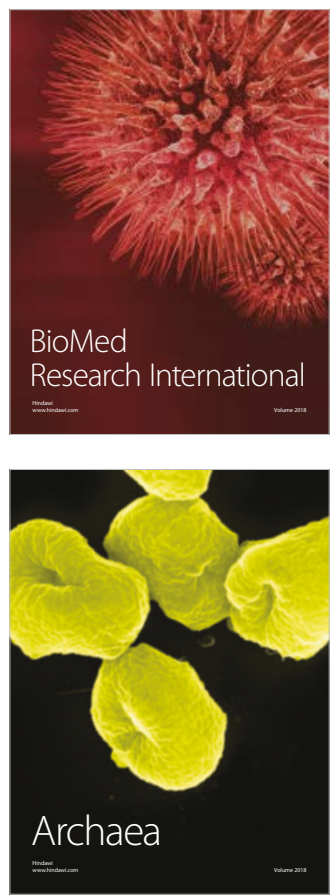